\title{
Differential phase-contrast BioCD biosensor
}

\author{
Ming Zhao, ${ }^{1}$ Wonryeon Cho, ${ }^{2}$ Fred Regnier, ${ }^{2}$ and David Nolte ${ }^{1}$ \\ 1Department of Physics, Purdue University, West Lafayette, Indiana 47907-2036, USA \\ 2Department of Chemistry, Purdue University, West Lafayette, Indiana 47907-2036, USA
}

*Corresponding author: zhaom@purdue.edu

Received 16 March 2007; accepted 22 May 2007;

posted 18 June 2007 (Doc. ID 80895); published 20 August 2007

\begin{abstract}
Common-path differential phase-contrast interferometry measures the spatial gradient of surface dipole density on a bio-optical compact disk (BioCD) and is sensitive to small changes in dipole density following molecular binding of target molecules out of solution. The recognition molecules are antibody IgG proteins that are deposited in periodic patterns on the BioCD using soft lithography or photolithography on the silanized silica surfaces of dielectric mirrors. Spatial carrier-wave sideband demodulation extracts the slowly varying protein envelope that modulates the protein carrier frequency. The experimental interferometric profilometry has surface height sensitivity down to $20 \mathrm{pm}$ averaged over a lateral scale of $70 \mu \mathrm{m}$ with a corresponding scaling mass sensitivity limit of $1.5 \mathrm{pg} / \mathrm{mm}$. Under the conditions of an IgG immunoassay with background changes caused during incubation, the scaling mass sensitivity is approximately $7 \mathrm{pg} / \mathrm{mm}$. A saturated reverse immunoassay performed with IgG at $100 \mathrm{ng} / \mathrm{ml}$ showed false positive and false negative rates of $0.2 \%$. (C) 2007 Optical Society of America
\end{abstract}

OCIS codes: $260.3160,170.1470$.

\section{Introduction to Spinning-Disk Interferometry}

In the past decade, solid-support protein microarray platforms have been developed as sensitive and multiplexed tools for the study of systematic interactions among proteins [1]. Many readout systems have been used to detect information from microarrays, including waveguides [2], optical fibers [3], surface plasmon resonance (SPR) [4], porous silicon [5], and ellipsometry [6], among others. Many of these approaches are label-free [7], including interferometric detection [8-10]. Interferometry working at the quadrature condition ( $\pi / 2$ difference between the reference and the probe beam) has high sensitivity to small changes in optical phase shift caused by the presence or absence of biomolecules, which is proportional to bound mass.

The key to stable interferometry is the ability to lock the phase difference between the reference and the signal beam at quadrature, unaffected by mechanical vibration or thermal drift. Common-path interferometry [11-14] is an established approach that accomplishes this phase-locking with an optical con-

0003-6935/07/246196-14\$15.00/0

(C) 2007 Optical Society of America figuration in which the signal and reference waves share a common path from the target to the detector. There are many ways to establish common-path interferometry that fall roughly into two categories: wavefront splitting and amplitude splitting configurations. Within the amplitude-splitting category are in-line approaches that use partial reflections at dielectric interfaces $[15,16]$. Within the wavefront splitting category are diffractive systems [17,18], including differential phase contrast techniques $[19,20]$ that we apply to the current class of phase-contrast bio-optical compact disk (BioCD).

We introduced the BioCD as a sensitive and scalable immunoassay platform that uses high-speed interferometric detection of biomolecules immobilized on a rapidly spinning disk [21]. The BioCD has two key attributes that separate this technology from other optical or interferometric biosensor technologies. The first is the intrinsic scalability of surfacenormal interferometric detection with the capacity for hundreds or thousands of assays per disk because the footprint per measurement can be as small as a square micrometer. The second is the high-speed laser scanning that sets the detection frequency far off low-frequency $1 / f$ noise with a $50 \mathrm{~dB}$ noise-floor suppression compared to static detection, allowing 
repeatable surface height measurements down to $20 \mathrm{pm}$. These two simple attributes provide the potential for high-speed label-free multianalyte assays with future applications in diagnostics, prognostics, and drug discovery.

There are several classes of BioCD distinguished by the way that stable quadrature interference is achieved. The microdiffraction (MD-class) BioCD was introduced first [22], which uses fabricated microstructures to lock the relative phase difference at $\pi / 2$. In this original class, 1024 gold spokes that have a height of $\lambda / 8$ are fabricated on a reflecting surface, and biomolecules are immobilized either on the gold spokes or on the land. The signal and the reference waves are both generated locally from a single optical mode (wavefront splitting), with the phase difference set and locked by the microstructure height, making it insensitive to mechanical motion or vibration of the apparatus. Immobilized biomolecules on the spokes or on the land change the relative phase between the two partial waves, and this phase is converted to intensity modulation through the quadrature interference condition.

A second class of BioCD, called the adaptive-optical class [23], uses self-adaptive nonlinear optical mixing in a photorefractive quantum well to adaptively lock the phase between the signal and the reference beam [24]. Patterned protein structures modulate the optical phase of the probe beam, which is sent through a photorefractive quantum well (PRQW) device that adaptively combines it with a local oscillator beam using two-wave mixing [25]. Two-wave mixing selfcompensates mechanical disturbances to maintain the quadrature condition with a compensation rate higher than $10 \mathrm{kHz}$. Phase modulation caused by the protein structure has frequencies higher than the compensation rate and is read out by a photodetector as amplitude modulation.

These first BioCD configurations (microdiffraction and adaptive optical) traded off complexity between the near field and the far field. In the microdiffraction BioCD, the disk structure was relatively complicated, with gold microstructures that needed specified sizes with tight tolerances. This was offset by relatively simple far-field detection that required only an apertured detector. The adaptive optical BioCD had the opposite tradeoff, with a very simple near-field structure on the disk, requiring no microfabrication other than protein patterning, but with a sophisticated adaptive mixer in the far field. Both of these BioCD configurations required complicated structures either in the near or in the far field.

In this paper, we introduce a third class of BioCD, the phase-contrast class, which dispenses with complex structures altogether, having simple disk fabrication in the near field and simple far-field detection. It is based on differential phase contrast profilometry [20,26-28], but with sensitivity to phase shifts caused by biomolecules immobilized on the surface. Because the protein surface profile is designable, we can select what patterns to make and detect. In particular, periodic patterns are amenable to sideband demodulation detection in which the periodicity provides a spatial carrier frequency (converted to temporal carrier frequency by rotating the disk), and the protein envelope function contains the information on the local height changes caused by specific binding of the analyte after an immunoassay. We describe the optical configuration of the disk and the role played by the condition of phase quadrature in the sensitive detection of protein profiles and derive analytical expressions with numerical simulations of the principles of operation. In Section 3 we give the experimental details of the optical configuration and the fabrication of the disks, followed in Section 4 by theoretical and numerical aspects of laser light scattering on spinning disks. Data and analysis are presented in Section 5 characterizing the metrological capabilities of the technique. The performance of a binary immunoassay is presented in Section 6 with a discussion of the detection limits of the phasecontrast system.

\section{Phase Contrast Detection of Spatially Varying Protein on Spinning Disks}

To achieve maximum interferometric sensitivity an interferometer must operate at or near the condition of phase quadrature at which the signal and the reference waves have a relative $\pi / 2$ phase difference. Under this condition, the detected intensity is linearly proportional to small phase modulation on the signal wave with maximum phase-to-intensity conversion efficiency. Differential phase contrast detection of protein patterned on a flat surface has a well-defined condition of quadrature defined by the path differences of a wave diffracted by a spatial variation in the overlying protein. This geometric condition is shown in Fig. 1, where a focused Gaussian beam of radius $w_{0}$ is incident on a reflecting flat surface (in this case a dielectric quarter-wave stack) with the optical axis (OA) incident on the discontinuous edge of an overlying protein layer. The spatial variation in the protein diffracts the wave into the far field. A ray originating from the protein side and a ray from the land side (the free surface is referred to as the "land") acquire a phase difference of $\pi / 2$ in the far field at the quadrature angle $\theta_{Q}$, given by

$$
\begin{aligned}
w_{0} \sin \theta_{Q} & = \pm \lambda / 4, \\
\theta_{Q} & = \pm \sin ^{-1}\left(\frac{\lambda}{4 w_{0}}\right) .
\end{aligned}
$$

There are two quadrature angles symmetric across the optic axis. The intensity change caused by the phase shift induced by the presence of the protein layer is equal but opposite at the opposite quadrature angles. Because the intensity shifts have equal magnitude, but opposite signs, a split photodetector with an inversion circuit is used to integrate the total intensity over each half of the response. If all the light were captured by a single-element photodetector, the opposite quadratures would cancel, and no protein 


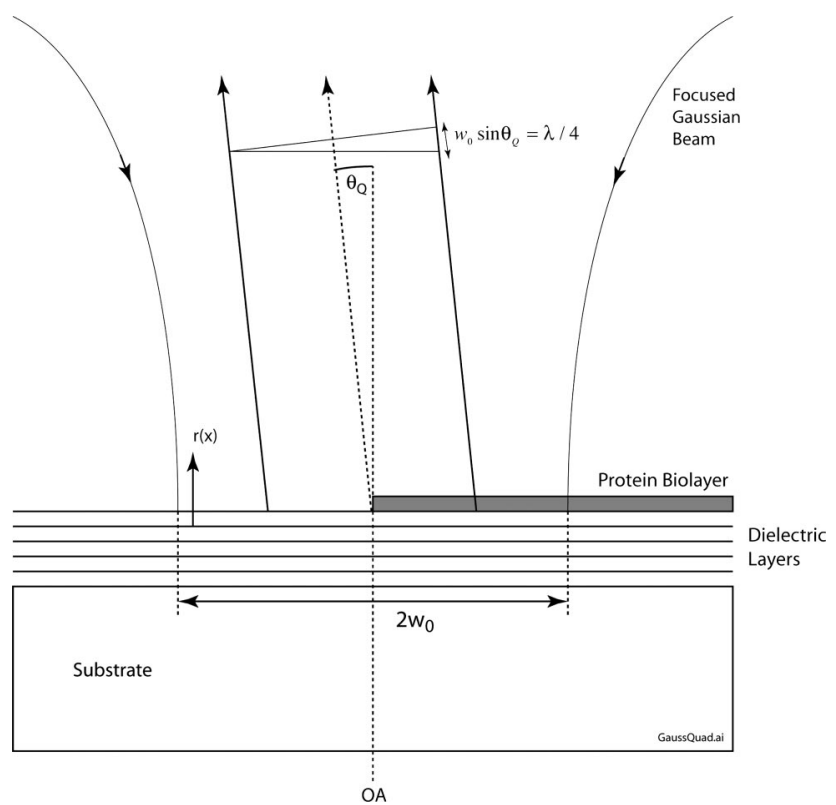

Fig. 1. Edge diffraction showing one quadrature angle for a biolayer on a high-reflectance dielectric stack with an antinode field condition at the top surface. The focused beam is Gaussian with a radius $w_{0}$. When the optic axis is at the edge of the biolayer, the quadrature angle is defined by a relative path difference to the far field of a quarter wavelength. There are two symmetric quadrature angles. Only one is shown.

would be visible. Similarly, if there are no variations in the protein density or thickness within the focused beam, no protein signal is detected. The protein detection by diffraction and the use of a split detector is therefore a differential measurement that detects spatial changes in protein density rather than the protein directly. The theoretical details of this differential detection are described in Section 3.

It is interesting to compare and contrast the detection of spatially patterned proteins on a flat surface with the conventional description of differential phase contrast as a surface metrology. The sensitivity of differential phase contrast detection is typically described as a slope sensitivity. This is because a slope on a reflecting surface simply deflects the Gaussian beam in the far field. The split detector in this case is described as a position-sensitive detector (PSD) and measures the deflection of the beam. Slope detection and protein detection are similar in the sense that both can be described by a Fraunhofer diffraction integral. However, a discontinuous protein step has no intrinsic length scale or slope, other than the length scale set by the radius of the probe beam. Furthermore, the far-field diffraction of a protein step is not strictly a Gaussian. Therefore, although the phase quadrature detection of protein shares much in common with slope detection, it differs in the details. Only when the protein profile changes slowly relative to the beam radius are the two descriptions the same.

The substrate under the biolayer plays a double role. First, it presents a flat and high-reflectance sup-

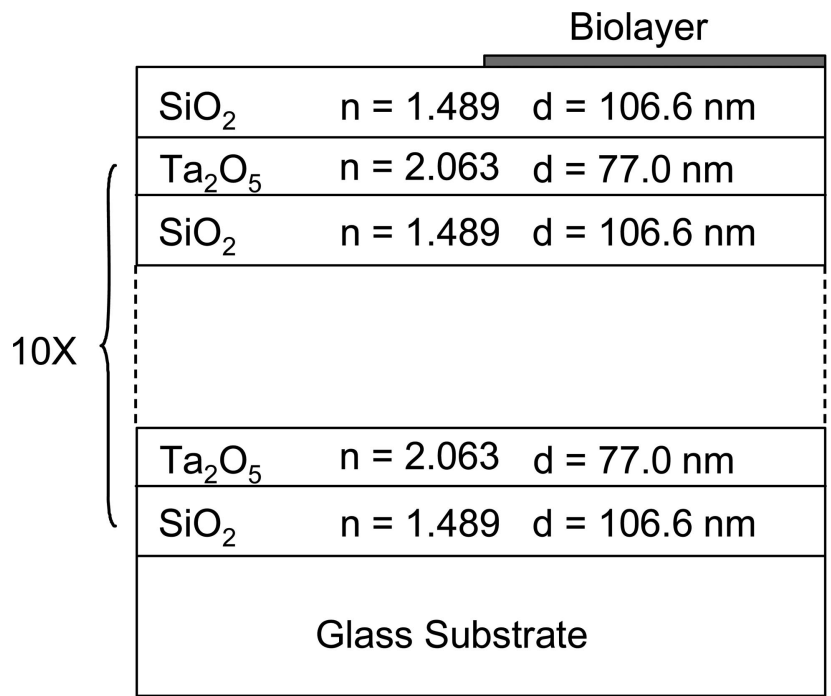

Fig. 2. Quarter-wave substrate dielectric mirror centered at a $633 \mathrm{~nm}$ wavelength that maximizes the biolayer phase shift by placing an electric field antinode at the stack surface.

port surface. The high reflectance provides for highly efficient photon detection, and the flatness sets the ultimate sensitivity for protein detection. The second role of the substrate is to provide the appropriate phase that maximizes the phase shift caused by the biolayer. The biolayer contributes a phase shift that is proportional to the magnitude of the local field at the layer. If the dielectric stack has an electric-field node at the surface, then the biolayer is "invisible" because there is no field to polarize the molecular dipoles. For a maximum phase change, it is necessary to construct a substrate with a field antinode at the surface. On a high-reflectance quarter-wave dielectric stack, this is commonly achieved by capping the stack with a low-index quarter-wave layer.

The substrate structure used in the calculations and experiments in this paper are shown in Fig. 2. The dielectric coating has ten repeat units of $\mathrm{Ta}_{2} \mathrm{O}_{5} / \mathrm{SiO}_{2}$ designed to have maximum reflection at $635 \mathrm{~nm}$ wavelength. The thickness for each dielectric layer is $\lambda / 4$, which is $77.0 \mathrm{~nm}$ for $\mathrm{Ta}_{2} \mathrm{O}_{5}$ and $106.6 \mathrm{~nm}$ for $\mathrm{SiO}_{2}$. On top of the dielectric stack is a $106.6 \mathrm{~nm}$ thick $\lambda / 4$ layer of $\mathrm{SiO}_{2}$, which makes the electric field at the surface a maximum. The dielectric stack rests on a $1.1 \mathrm{~mm}$ thick $100 \mathrm{~mm}$ diameter Schott Borofloat Pyrex glass substrate with a $15 \mathrm{~mm}$ diameter hole in the middle to fit the spinner.

\section{Laser Light Scattering on a Spinning Disk}

In the theoretical derivation of phase-contrast quadrature detection of thin biolayers, we start with an idealized perfectly reflecting planar surface carrying a protein layer of thickness $h(x)$. No explicit boundary conditions are assumed for the surface other than the reflected phase. Consider the normalized twodimensional intensity distribution of an incident Gaussian beam, 


$$
I(\rho)=\frac{1}{2 \pi w_{0}^{2}}\left(e^{-\rho^{2} / 2 w_{0}{ }^{2}}\right),
$$

where $\rho^{2}=x^{2}+y^{2}$ with corresponding dimensionless electric field

$$
g(\rho)=g(x, y)=\frac{1}{\sqrt{2 \pi} w_{0}} e^{-\rho^{2} / 4 w_{0}{ }^{2}},
$$

and two-dimensional Fourier transform

$$
G\left(k_{x}, k_{y}\right)=2 \sqrt{2 \pi} w_{0} e^{-w_{0} 2 k^{2}} .
$$

The two-dimensional diffraction problem is considered in the Fraunhofer regime. The modulated near field is

$$
\begin{aligned}
E(x, y) & =g(x, y) \exp \left(i \phi_{1} h(x-v t, y)\right) \\
& \approx g(x, y)\left[1+i \phi_{1} h(x-v t, y)\right],
\end{aligned}
$$

where the surface topology, including the motion of the disk, is contained in the real-valued height function $h(x-v t, y)$, and the constant $\phi_{1}$ carries the phase information related to the refractive index of the surface material. This equation is valid for small phase and amplitude modulation.

The far field is

$$
\begin{aligned}
E\left(k_{x}, k_{y}\right) & =G\left(k_{x}, k_{y}\right)+i \phi_{1} F T[g(x, y) h(x-v t, y)] \\
& =G\left(k_{x}, k_{y}\right)+i \phi_{1} H(k, t)
\end{aligned}
$$

where FT stands for Fourier transform and the transformed function is

$$
H(k, t)=F T[g(y, x) h(y, x-v t)] .
$$

For a nodal boundary condition $\phi_{1}=0$ to lowest order, meaning that a biofilm on a nodal surface causes no phase shift and is hence invisible. For an ideal antinodal surface (the case considered in this paper), it takes on a maximum value. These two limiting cases are

$$
\phi_{1}= \begin{cases}0 & \text { nodal } \\ \frac{4 \pi\left(n_{p}^{2}-1\right)}{\lambda} & \text { antinodal }\end{cases}
$$

with general substrates having values between these extremes.

The intensity at the detection (Fourier) plane is

$$
\begin{aligned}
I\left(k_{x}, k_{y} ; t\right)= & \left|G\left(k_{x}, k_{y}\right)+i \phi_{1} H(k, t)\right|^{2} \\
\approx & \left|G\left(k_{x}, k_{y}\right)\right|^{2}+i G\left(k_{x}, k_{y}\right)\left[\phi_{1} H(k, t)\right. \\
& \left.-\phi_{1}^{*} H^{*}(k, t)\right] \\
= & \left|G\left(k_{x}, k_{y}\right)\right|^{2}+2 G\left(k_{x}, k_{y}\right) \operatorname{Im}\left(\phi_{1} H(k, t)\right) .
\end{aligned}
$$

The detected photocurrent is obtained by integrating Eq. (7) over the Fourier-plane response function $R(k)$ that can be controlled by appropriate apertures or split detectors. The normalized photocurrent is

$$
i_{d}(t)=\int_{-\infty}^{\infty} R\left(k_{x}, k_{y}\right) I\left(k_{x}, k_{y} ; t\right) \mathrm{d}^{2} k .
$$

Restricting the problem to the one-dimensional case, the differential phase-contrast signal in the $x$-direction is obtained using a split detector. The photocurrent for a differenced-split detector is

$$
\begin{aligned}
i_{d}(t) & =\int_{-\infty}^{0} I\left(k_{x}, t\right) \mathrm{d} k_{x}-\int_{0}^{\infty} I\left(k_{x}, t\right) \mathrm{d} k_{x} \\
& =4 \int_{0}^{\infty} G\left(k_{x}\right) \operatorname{Im}\left(\phi_{1} F\left(k_{x}, t\right)\right)_{o d d} \mathrm{~d} k_{x},
\end{aligned}
$$

where $R\left(k_{x}\right)$ is now a step function at $k_{x}=0$, and the subscript "odd" refers to only the odd functions of $\operatorname{Im}\left(\phi_{1} F(k, t)\right)$ because $G(k)$ is already an even function of $k$. The detector current is proportional to the quantity

$$
i_{d}=\frac{1}{2} \phi_{1} \frac{|g(x)|^{2}}{|g(0)|^{2}} \otimes\left[\frac{\mathrm{d} h(x)}{\mathrm{d} x}+\frac{1}{6} \frac{\mathrm{d}^{3} h(x)}{\mathrm{d} x^{3}}+\cdots\right],
$$

which also illustrates the contribution from higherorder derivatives. In the simplest case, for slowly varying $h(x)$ relative to $g(x)$, and higher-order terms neglected, this gives

$$
i_{d}(t)=\frac{1}{2} \phi_{1}\left[\frac{I(x)}{I(0)} \otimes \frac{\mathrm{d} h(x-v t)}{\mathrm{d} x}\right],
$$

which is proportional to the first derivative of the surface height convolved with the normalized beam intensity. Because of the convolution, any surface protein density fluctuation with a length scale smaller than the incident beam waist is averaged over.

The change in the far-field intensity caused by protein on the surface is given by the second term in Eq. (7) and is displayed in Fig. 3. The far-field pattern is asymmetric across the optic axis regardless of the position of the protein edge relative to the optic axis in the near field. The second curve in Fig. 3 is the diffraction pattern when the protein edge is one beam radius away from the optic axis. The higherspatial frequency oscillations are proportional to the offset, and the overall amplitude is smaller, but the pattern is still asymmetric and is detected by integrating and differencing over the split photodetector, as in Eq. (8). 


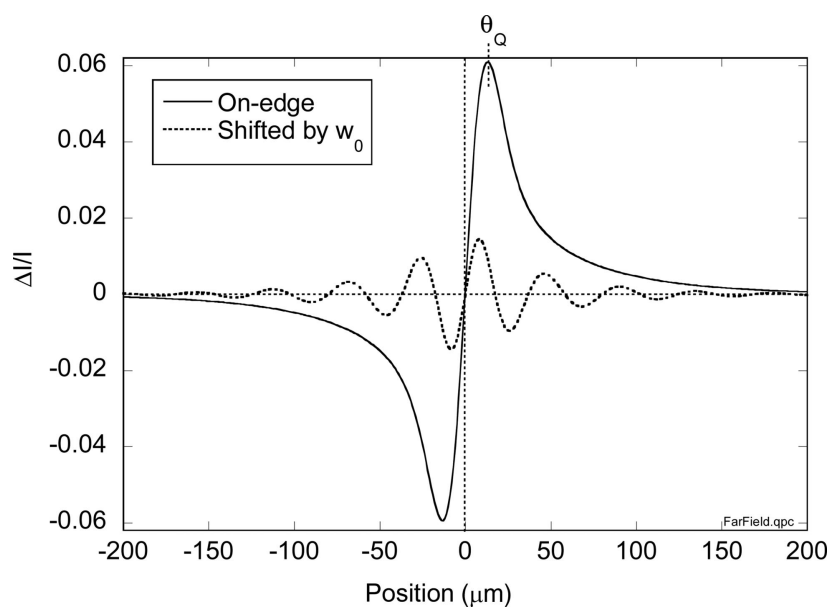

Fig. 3. Calculated far-field diffracted intensity changes from a $6 \mathrm{~nm}$ biolayer with $n_{p}=1.4$ on a dielectric stack for two different locations of the optic axis relative to the protein edge at $x=0$ and at $x=w_{0}$.

\section{A. Wide Protein Ridges}

A protein ridge and its differential response are simulated in Fig. 4. The refractive index for the protein is assumed to be $n_{p}=1.4$ with a height of $h_{0}=6 \mathrm{~nm}$. The phase shift upon reflection from the antinodal surface is taken to be $\Delta \phi=4 \pi\left(n_{p}{ }^{2}-1\right) h_{0} / \lambda=0.11$. The full width of the laser spot is $2 w_{0}=24 \mu \mathrm{m}$. Because the response is differential, a gradual slope on the edge of the printed protein reduces the magnitude of the phase-contrast signal. This is illustrated in the figure by increasing the slope width of the protein ridge from 1 to $50 \mu \mathrm{m}$ for a $128 \mu \mathrm{m}$ wide ridge. The simulated protein profile is composed of two Fermi functions,

$$
h(x, t)=h_{0} f\left[\left(x-\left(x_{L}-v t\right)\right) / w\right] f\left[\left(x-\left(x_{R}-v t\right)\right) / w\right],
$$

where $f(x)$ is the Fermi function, the width is set by the slope-width parameter $w$, and the width of the protein spoke is $x_{L}-x_{R}$. The total protein remains constant under all profiles. The resulting differential phase-contrast image along the scan direction is shown in Fig. 4(b). The positive peak is from the leading edge, and the negative peak is from the trailing edge of the protein ridge. For $n_{p}=1.4$ and a $6 \mathrm{~nm}$ height, the maximum modulation for the sharp edge profile is $5.7 \%$ and decreases with increasing slope width. The half-response point occurs when the slope width in the Fermi function is $w=w_{0}$. The response is given by the convolution of the Gaussian with the exponential edge of the Fermi function

$$
\frac{\Delta I}{I}=\frac{2 \pi\left(n_{p}^{2}-1\right)}{1+\left(w / w_{0}\right)^{2}} \frac{h_{0}}{\lambda}=\frac{\mathrm{d} S}{\mathrm{~d} h} \frac{h_{0}}{1+\left(w / w_{0}\right)^{2}} .
$$

This function is linear in the protein height $h$ and is plotted in Fig. 5, showing the knee when $w=w_{0}$. For a sharp protein edge with $n_{p}=1.4$, the conversion

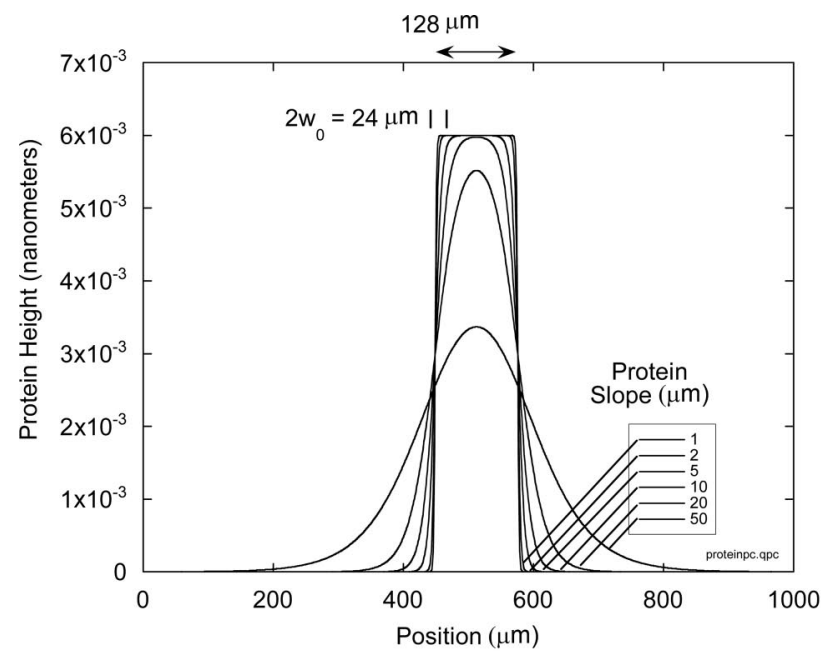

(a)

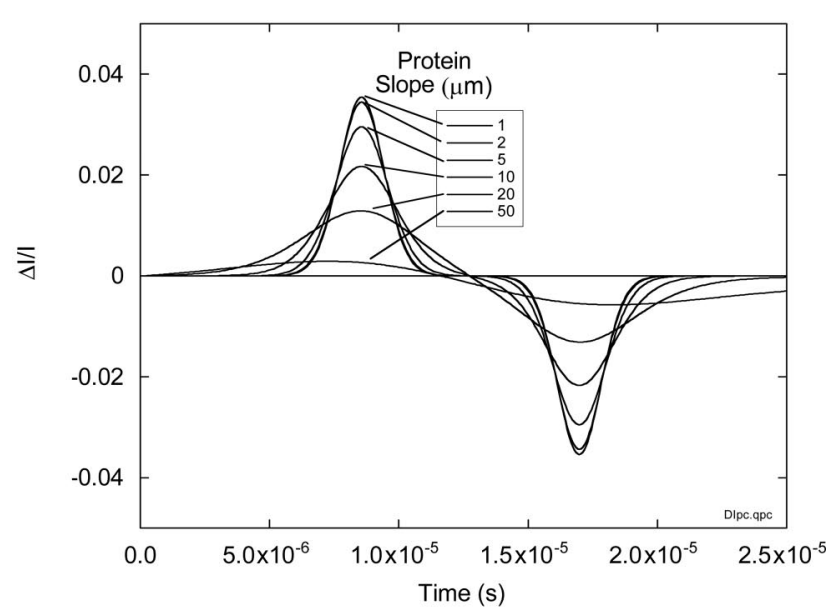

(b)

Fig. 4. Simulated protein profile in (a) and the calculated phasecontrast response to the spinning disk in (b). The beam radius is $12 \mu \mathrm{m}$ and the protein spoke width is $128 \mu \mathrm{m}$. The curves show the effect of increasing slope widths from 1 to $50 \mu \mathrm{m}$.

from protein height to modulation is

$$
\frac{\mathrm{d} S_{\text {edge }}}{\mathrm{d} h}=\frac{2 \pi\left(n_{p}{ }^{2}-1\right)}{\lambda}=0.95 \% / \mathrm{nm}
$$

under the conditions of nearly $100 \%$ reflectance and with an electric-field antinode at the disk surface. Other substrates and field conditions have different conversion factors.

\section{B. Sinusoidal Protein Profile}

A second special case of interest is when the protein takes on a sinusoidal profile given by

$$
h(x, t)=\frac{h_{0}}{2} \sin \left(k_{0}(x-v t)\right),
$$

where $h_{0}$ is the full protein height. The far field, from Eq. (5), is 


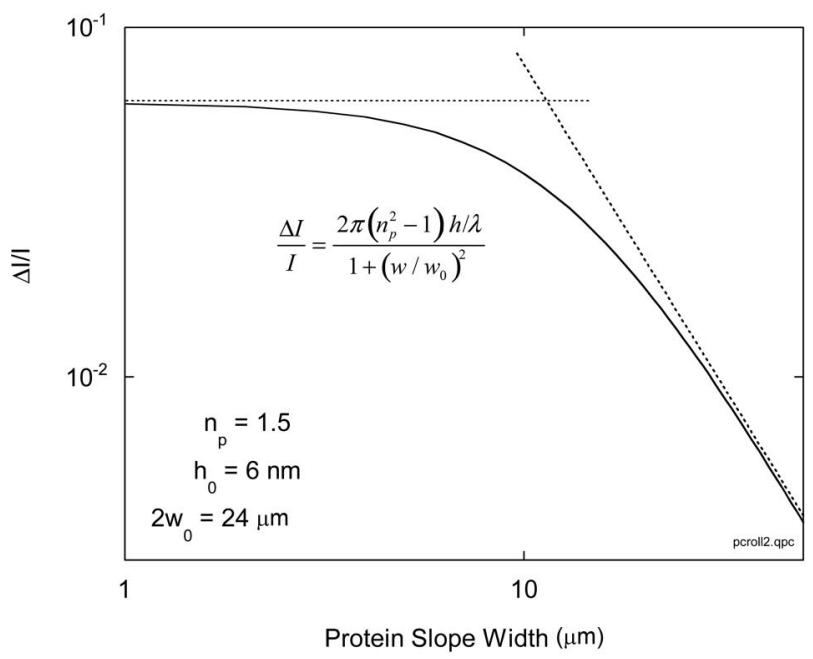

(a)

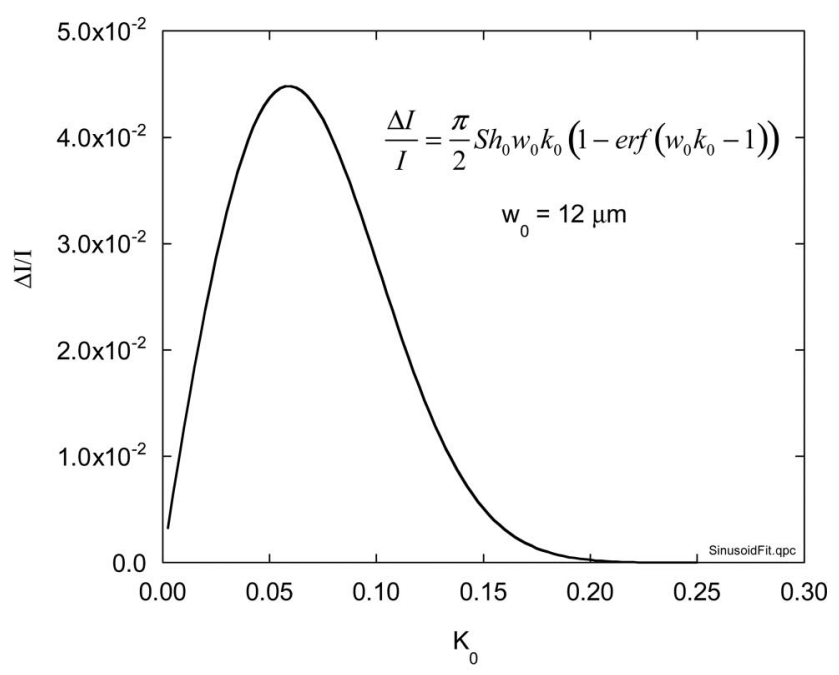

(b)

Fig. 5. (a) Theoretical phase-contrast response to a protein ridge expressed as relative intensity modulation as a function of the protein slope width. The knee occurs when the protein width is comparable to the beam radius. (b) Theoretical phase-contrast response to a sinusoidal protein profile expressed as relative intensity modulation as a function of spatial frequency. The response has a maximum at $w_{0} k_{0}=1$.

$E(k)=G(k)+i \frac{1}{2} \phi_{1} h_{0} F T\left[g(x) \sin \left(k_{0}(x-v t)\right)\right]$.

The expression for the Fourier transform is obtained in analytical form as

$$
\begin{aligned}
H= & F T\left[g(x) \sin \left(k_{0}(x-v t)\right)\right] \\
= & \frac{i}{\sqrt{2}} g(0)\left[\exp \left(i k_{0} v t-w_{0}^{2}\left(k+k_{0}\right)^{2}\right)\right. \\
& \left.-\exp \left(-i k_{0} v t-w_{0}^{2}\left(k-k_{0}\right)^{2}\right)\right],
\end{aligned}
$$

to give the far field

$$
\begin{aligned}
E(k)= & G(k)+\frac{\phi_{1} h_{0}}{2 \sqrt{2}} g(0)\left[\exp \left(i k_{0} v t-w_{0}^{2}\left(k+k_{0}\right)^{2}\right)\right. \\
& \left.-\exp \left(-i k_{0} v t-w_{0}^{2}\left(k-k_{0}\right)^{2}\right)\right]
\end{aligned}
$$

with the far-field intensity given by

$$
\begin{aligned}
I(k)= & |E(k)|^{2} \\
= & \left.|G(k)|^{2}+\phi_{1} h_{0} g(0) \sin \left(k_{0} v t\right) e^{-w_{0}^{2}\left(k^{2}+k_{0} 2\right.}\right) \\
& \times \sinh \left(2 w_{0}{ }^{2} k k_{0}\right) .
\end{aligned}
$$

This intensity is detected by the split detector function, producing the relative modulation

$$
\begin{aligned}
\frac{\Delta I(t)}{I} & =2 \phi_{1} h_{0} \sin \left(k_{0} v t\right) \int_{0}^{\infty} e^{-w_{0}^{2}\left(k^{2}+k_{0}^{2}\right)} \sinh \left(2 w_{0}^{2} k k_{0}\right) \mathrm{d} k \\
& =\frac{\pi}{2} \frac{\mathrm{d} S}{\mathrm{~d} h} h_{0} w_{0} k_{0}\left(1-\operatorname{erf}\left(w_{0} k_{0}-1\right)\right) \sin \left(k_{0} v t\right),
\end{aligned}
$$

which increases linearly with spatial frequency $k_{0}$ at low values relative to $1 / w_{0}$ because of the sensitivty of phase contrast to slope, and rolls off at high spatial frequency when the corrugation no longer can be resolved by the focal spot size. The magnitude of the intensity modulation as a function of $k_{0}$ is shown in Fig. 5(b). The maximum sensitivity occurs at $w_{0} k_{0}=1$ with an amplitude (half peak-to-peak) of $\Delta I /\left.I\right|_{\max }=\pi^{2}\left(n_{p}{ }^{2}-1\right) / 2 \lambda\left(h_{0}\right)$. As an example, for $n_{p}=1.4, \lambda=635 \mathrm{~nm}$, and $h_{0}=6 \mathrm{~nm}$ for $w_{0} k_{0}=1$, the amplitude is $\Delta I / I=4.5 \%$, and the conversion from protein height to modulation is

$$
\frac{\mathrm{d} S_{\sin }}{\mathrm{d} h}=\frac{\pi^{2}\left(n_{p}^{2}-1\right)}{2 \lambda}=0.75 \% / \mathrm{nm}
$$

under the conditions of nearly $100 \%$ reflectance and a field antinode at the surface.

\section{Disk Patterning and Optical Detection}

Phase-contrast detection detects the gradient of protein density. Therefore, optimal detection efficiency requires the protein to have a spatial frequency approximately equal to the inverse focal spot width. Patterning of protein is performed by several different methods, including photolithography, a gel-stamp printing method, and protein spotting. The surface immobilization chemistries are either covalent or physical adsorption.

Photolithography follows a standard protocol for photoresist patterning. A disk is coated with AZ 1518 photoresist and spun at $3000 \mathrm{rpm}$ for $50 \mathrm{~s}$ to form a uniform layer of photoresist about $20 \mu \mathrm{m}$ thick. It is soft-baked at $90^{\circ} \mathrm{C}$ for $15 \mathrm{~min}$ to remove organic solvents in the photoresist. The disk is cooled in the air to room temperature and exposed by near-UV light through a patterned photomask in a Canon PLA 
$501 \mathrm{~F}$ mask aligner. The exposed disk is developed in 1:1 AZ Developer and washed in DI Water for $30 \mathrm{~s}$.

We use two types of surface chemistries for photolithographic patterning of proteins. The first is a polysuccinamide (PSI) polymer coating conjugated with biotin. After photolithography the disk is covered by $10 \mathrm{~mL}$ of $10 \mu \mathrm{g} / \mathrm{mL}$ avidin in $\mathrm{pH} 8.510 \mathrm{mM}$ HEPES buffer with $160 \mathrm{mM} \mathrm{NaCl}$. Biotin on the exposed surface binds the avidin. The photoresist is then removed by acetone, and the disk covered again with $10 \mathrm{~mL}$ of biotinylated antibody solution at a concentration of $10 \mu \mathrm{g} / \mathrm{mL}$ in $\mathrm{pH} 7.410 \mathrm{mM}$ phosphate buffered saline (PBS). The antibodies bind covalently to the patterned avidin.

A different surface chemistry for photolithography uses a (3-animopropyl)triethoxysilane (APTES) coating that is conjugated with poly(ethylene glycol) diglycidyl ether. For this coating, after photolithography the disk is covered by $10 \mathrm{~mL}$ of $1 \%$ sodium borohydride in DI water for $24 \mathrm{~h}$ at room temperature, deactivating the exposed surface. The photoresist is removed by acetone, and a protein solution (antibody or IgG) at $10 \mu \mathrm{g} / \mathrm{mL}$ in PBS buffer is applied to the disk. The surface previously covered by photoresist remains active and covalently binds the protein, while the previously exposed surface is passivated.

Although photolithography produces well-defined patterns, which is ideal for phase-contrast detection, we also used soft lithography based on gel stamping, which is low cost and convenient, shown in Fig. 6(b). A polyacrylamide gel is made in a $10 \mathrm{~mL}$ mixture (consisting of $4 \mathrm{~mL} 30 \%$ 37.5:1 acrylamide/bis acrylamide, $2.5 \mathrm{~mL} 1.5 \mathrm{M}$ Tris/HCl Buffer at $\mathrm{pH} 8.8,3.3$ $\mathrm{mL}$ of DI water and $200 \mu \mathrm{L} 10 \%$ ammonium persulfate). Protein is dissolved in this mixture at a concentration of $10 \mu \mathrm{g} / \mathrm{mL}$, and $20 \mu \mathrm{L}$ of TEMED is added to the mixture after degassing to catalyze the polymerization. The mixture is immediately poured into a mold that was previously fabricated out of $50 \mu \mathrm{m}$ thick SU8 photoresist. After $30 \mathrm{~min}$, the polymerization of acrylamide is complete, and the gel is lifted

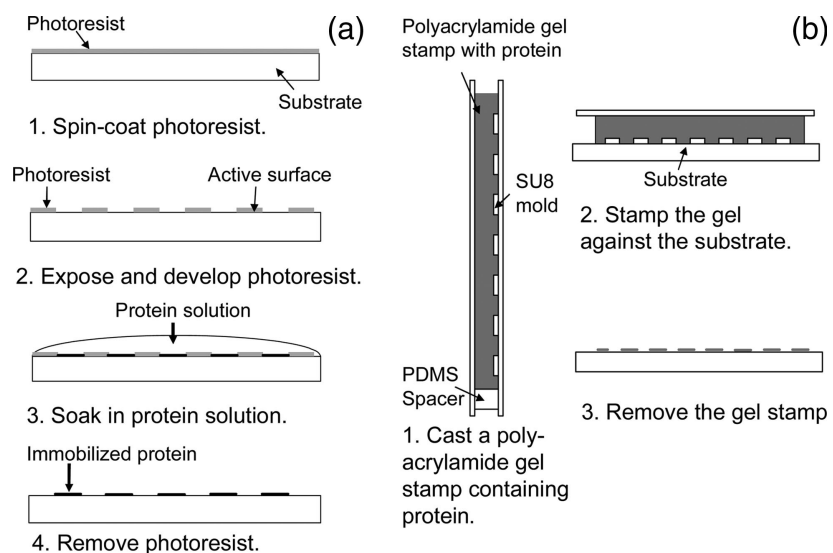

Fig. 6. Schematics of the patterning of protein. (a) Photolithography. Photoresist is patterned on the substrate surface, which is exposed to protein solution to immobilize protein. (b) Gel stamping. A patterned polyacrylamide gel with protein is made and pressed against a functionalized substrate.

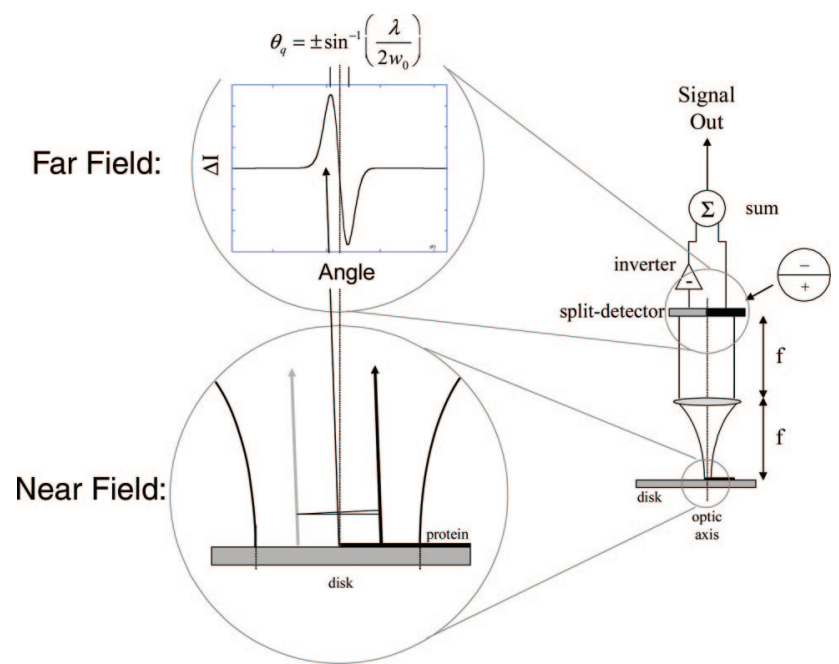

Fig. 7. (Color online) Optical configuration and layout for differential phase-contrast detection of protein patterns on the spinning disk. Far-field intensity modulation is antisymmetric. A split photodetector with relative inversion converts the asymmetry into a signal that is proportional to the protein height. Photodetector has four quadrants that can be combined as the sum, left-right difference, or up-down difference.

carefully from the mold. The gel retains the pattern of the mold, and a surface-functionalized disk is pressed against the gel stamp for $30 \mathrm{~min}$. Protein diffuses out of the gel at the contact interface and is captured onto the surface of the disk. Gel printing can be used with multiple surface chemistries, but usually it is used with physical adsorption through silanization of the silica surface. The silanization follows a standard protocol in which the disk is soaked in $10 \mathrm{mM}$ chlorodimethyloctadecylsilane in toluene for $18 \mathrm{~h}$. Protein is adsorbed by hydrophobic interaction by the methyl end group of the coating.

The optical detection system for the phase-contrast BioCD is shown in Fig. 7. A $635 \mathrm{~nm}$ diode laser beam is focused by a $5 \mathrm{~cm}$ focal-length objective lens to a $20 \mu \mathrm{m}$ diameter spot on a disk that is mounted on a stable spinner (Lincoln Laser, Inc.) and is spun at $80 \mathrm{~Hz}$. The reflected and diffracted light is collected by the same objective lens and directed to a quadrant photodetector by a beam splitter. Two lenses that have a focal length of $1.8 \mathrm{~cm}$ and $5 \mathrm{~cm}$, respectively, transform the beam to the detector placed at the Fourier plane of the system. The quadrant detector has three output channels: the total intensity, the difference between left and right, and the difference between top and bottom. One of the difference channels gives the phase contrast signal, while the other serves as a diagnosis of system alignment and disk wobble. The total intensity channel provides amplitude information related to Rayleigh and other scattering losses, which are small compared to the phase-contrast signal produced by the protein pattern.

The disk is scanned in successive tracks of increasing radius. The signal from the quadrant detector is collected by an oscilloscope that is triggered by a synchronization signal from the spinner controller 


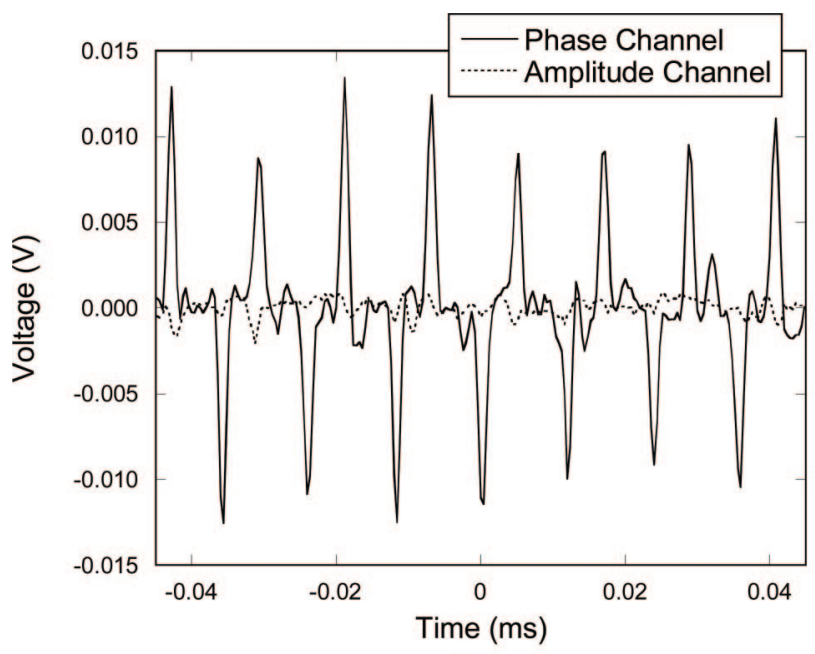

(a)

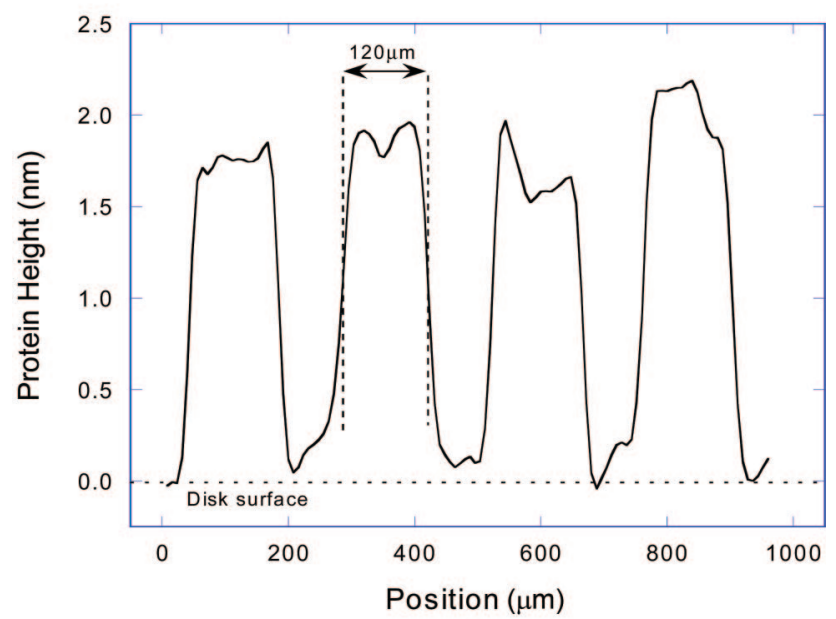

(b)

Fig. 8. (Color online) (a) Time trace of the differential phasecontrast channel in response to four protein ridges passing through the focused laser spot. The leading edge causes a negative signal while the trailing edge causes a positive signal. (b) Integrated differential signal expressed in terms of protein height. Irregularities are not noise, but are repeatable, and hence real, structures related to the protein printing.

that corresponds to a set orientation of the disk. The acquired time trace for a pattern of biotinylated antirabbit attached to avidin by photolithography is shown in Fig. 8(a). The differential signal produced by stepping up and down the protein spokes is clearly seen in the phase channel, while the amplitude channel remains almost flat, indicating little scattering loss. The surface protein profile is obtained by integrating the signal from the phase-contrast channel and removing low-frequency drift, as shown in Fig. 8(b). The conversion from detector voltage output to height is made using the theoretical conversion factor from Eq. (13). The protein profile in Fig. 8(b) is the true protein profile convolved with the point-spread function of the laser beam. The beam radius for these data was approximately $20 \mu \mathrm{m}$, and the protein ridge width was $120 \mu \mathrm{m}$. The irregularities in the protein profiles are not noise, but are repeatable upon repeated scans. They are real structures associated with the protein patterning process.

The time traces acquired at different radii are combined into a two-dimensional (2D) data array and plotted as a 2D differential surface topology, as shown in Fig. 9. In this two-dimensional visualization, structured protein can be easily distinguished from surface defects such as dust or salt deposits. Figure 9(a) shows a 2D topology of a disk that was gel printed with bovine serum albumin (BSA) in a pattern consisting of radial ridges that has an angular spatial frequency of $1024 / 2 \pi$. Figure 9(b) shows photolithographically patterned avidin on a disk coated with PSI biotin. The photolithographic disk was patterned with a checkerboard pattern of radial spokes. The pattern is divided into $321 \mathrm{~mm}$ wide bands radially, and each band is divided into 16 angular segments. Half of the segments are patterned with 64 radial spokes that have an angular spatial frequency of $1024 / 2 \pi$. Following each patterned segment is a blank segment serving as a reference surface, which is the bare PSI coating. The radial scanning pitch $(\Delta r)$ separating tracks is $20 \mu \mathrm{m}$. The leading edge of the protein spokes is bright and the trailing edge dark, corresponding to the stepup and stepdown of the laser beam from the printed protein. The measured protein height is between 1 and $2 \mathrm{~nm}$ for both cases, suggesting submonolayer surface protein coverage.

The two orthogonal output channels from the quadrant photodetector provide a means to obtain the isotropic modulus of the differential surface topology. The up-down channel can be added in quadrature with the left-right channel. This is shown in Fig. 9(c) for two irregular protein spots printed onto a dielectric disk. The A-channel is left-right, and the B-channel is updown. These two orthogonal images are added in quadrature, and the third image shows relatively uniform rings around the protein spots, as well as small rings around the dust or defect spots in the data. The modulus image is unbiased with respect to the spin direction. For assays, we rely on radial spoke patterns, so only one of the two channels needs to be acquired.

The phase-contrast detection system is calibrated against the adaptive-optical class detection system [23]. The adaptive-optical system directly measures optical phase shifts and is calibrated by a $5 \mathrm{MHz}$ electro-optic phase modulator that produces a known phase modulation. A photolithography-patterned antinodal (for $835 \mathrm{~nm}$ wavelength) dielectric disk printed with avidin was scanned in both systems with the same laser wavelength at $835 \mathrm{~nm}$, and the scanning results were compared. A region of the disk where the protein printing was most uniform was chosen for the comparison. When scanned by the adaptive optical detection system, an average phase difference of $0.069 \mathrm{rad}$ between the land and the avidin spokes was observed. This corresponds to an average protein height of $3.6 \mathrm{~nm}$ for an avidin refractive index of $n_{p}=1.4$. In phase-contrast scanning, a $47 \mathrm{pf}$ capacitor serves as a high-pass filter to remove the 


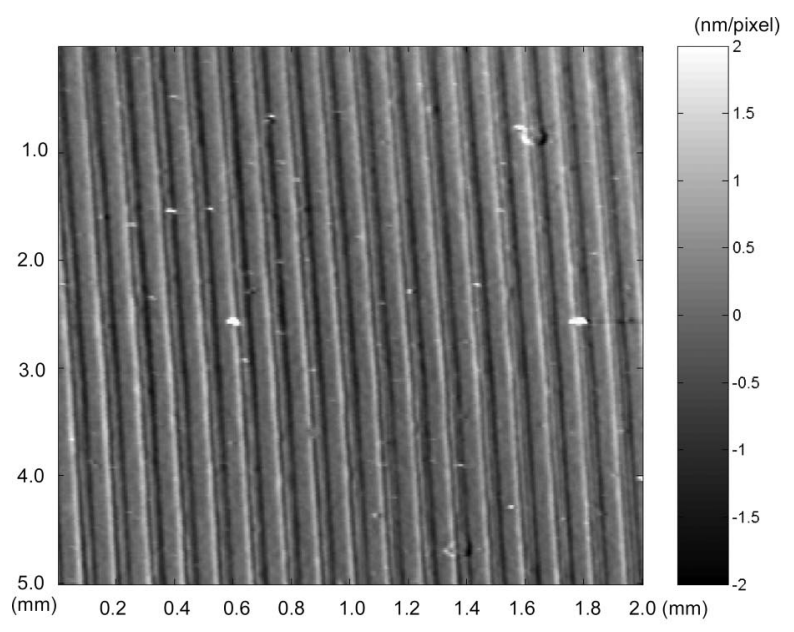

(a)

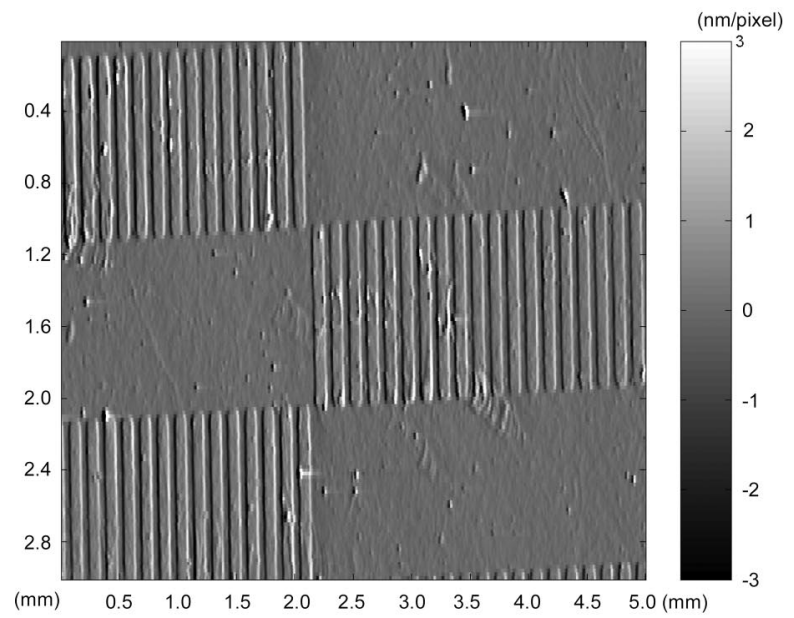

(b)
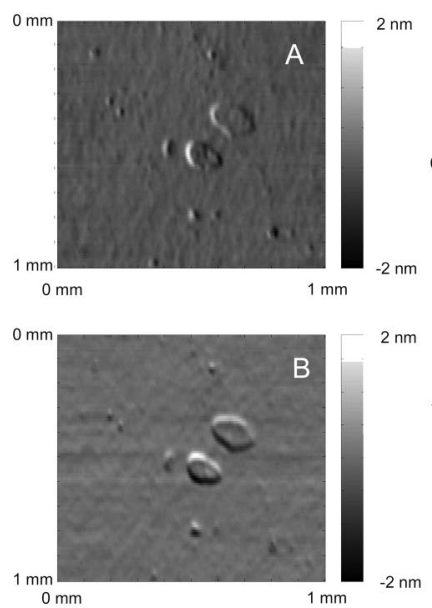

(c)

Fig. 9. Examples of phase-contrast detection of protein patterns. Pattern in (a) is gel-printed protein spokes on a silanized surface. Pattern in (b) is part of a checkerboard disk patterned by photolithography on APTES surface. (c) An example of adding the two phase-contrast channels in quadrature to extract the modulus differential topology of protein on a disk. The A-channel is the leftright detector channel, and the B-channel is the up-down detector channel. The combined channel shows uniform edge detection around the printed protein spots. slow drift caused by disk wobble, which reduced the detected signal by a factor of 2.9. The phase-contrast scan showed an average intensity modulation of $0.8 \%$ by a single edge, which converts to $2.32 \%$ without the high-pass filter. The resulting experimentallycalibrated sensitivity is $d S / d h=2.32 \% / 3.6 \mathrm{~nm}=$ $0.64 \% / \mathrm{nm}$, compared with the theoretical value of $0.95 \% / \mathrm{nm}$ from Eq. (13) that assumes that the protein edge is perfectly sharp. However, the comparison cannot be direct because phase-contrast scanning detects the derivative of the optical phase shift, while the adaptive-optical class that detects the optical phase shift directly. The discrepancy can be resolved if the protein edge is not sharp, but increases over a length of about $10 \mu \mathrm{m}$, which is below the spatial resolution of the probe spot. Therefore, within the ability of the laser scanning to resolve spatial variations, the theoretical and the experimental values are in close agreement.

\section{Sideband Demodulation}

One of the advantages of immobilizing protein with a high spatial-frequency pattern is the use of Fourier analysis to distinguish periodic protein signals from background noise. Figure 10 shows the power spectrum acquired by a spectrum analyzer of a disk photolithographically patterned with 1024 periodic radial spokes of avidin-captured biotinylated antirabbit IgG. The resolution bandwidth is $3 \mathrm{kHz}$. The system is operating well above the laser and electronic noise of the system, and well beyond $1 / f$ noise. The spectral power density of the protein signal is $-40 \mathrm{~dB}$, corresponding to $1 \%$ intensity change caused by the protein spokes, which is $25 \mathrm{~dB}$ above the noise floor of the disk roughness.

The BioCD can be segmented into numerous equivalent areas or "wells" for multiple assay applications. An example of the power spectrum averaged over a segment covering an area of $90 \mathrm{~mm}^{2}$, or about $1 / 64$ th of the disk, is shown in Fig. 10(b) for biotinylated antibodies bound to printed avidin spokes. The power signal-to-noise ratio for this "well" is 400:1 with a baseline roughness of $100 \mathrm{pm}$. The equivalent protein height and surface roughness are calculated using the signal power and the conversion factor of Eq. (13).

The spatially patterned protein structure produces a signal at a carrier frequency with a modulation envelope that is proportional to the local height of the printed protein structure. This is similar to radio reception in which the signal is carried by the envelope of a high-frequency carrier wave. We use sideband demodulation to remove the carrier frequency and reveal only the local envelope of the protein carrier. A 2D differential topology [shown in Fig. 11(a)] is transformed into the Fourier domain, and the position of the first Fourier component of the patterned structure is identified. The Fourier component together with its neighboring region is shifted to zero frequency and transformed back into the space domain. This demodulation process serves as a bandpass filter, which picks up only signals that have 


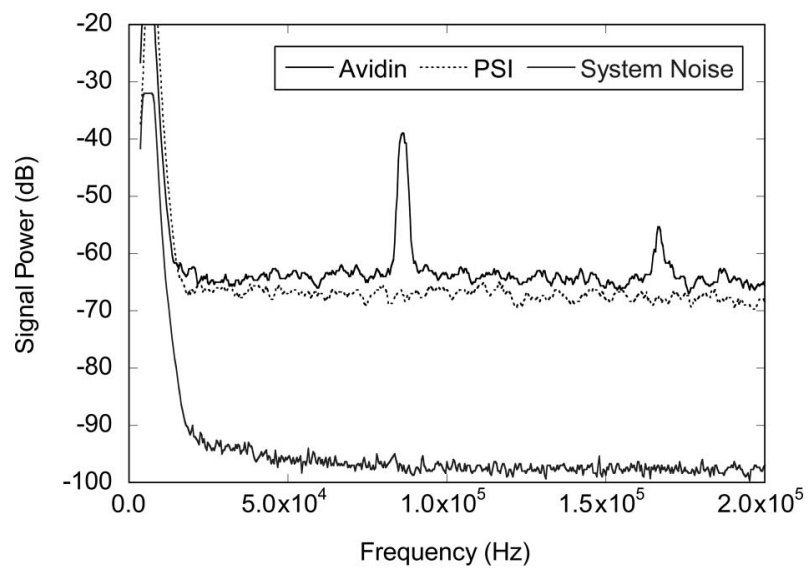

(a)

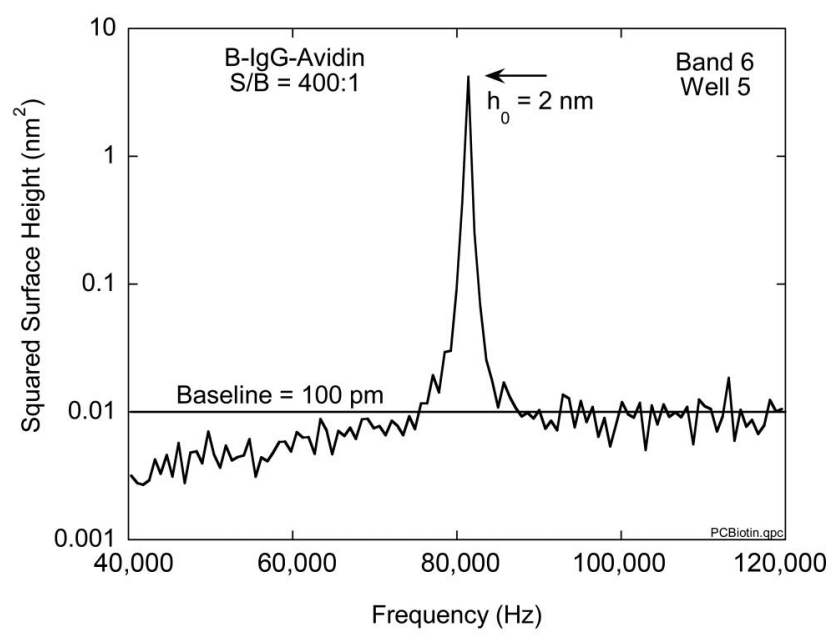

(b)

Fig. 10. (a) Comparison of the power spectra of printed protein ridges, bare functionalized disk, and system noise at $3 \mathrm{kHz}$ bandwidth. Surface roughness contributes $30 \mathrm{~dB}$ of noise when the disk spins. Protein signal is $25 \mathrm{~dB}$ above the spinning-disk noise floor. High-frequency detection on the spinning disk suppresses the noise floor by $50 \mathrm{~dB}$ relative to $1 / f$ noise at dc. (b) Power spectrum of 1024 avidin/biotin spokes printed photolithographically. Disk was spinning at $80 \mathrm{~Hz}$, high-pass filtered, and averaged over a disk segment covering $1 / 64$ th of the disk surface with a $3 \mathrm{kHz}$ bandwidth. The power signal-to-baseline is 400:1. The baseline height of $100 \mathrm{pm}$ measures the mean-squared surface roughness of the disk.

spatial frequencies close to the periodic protein pattern. In the figure, the lower and upper cutoff angular spatial frequencies are $2 \pi / 600$ and $2 \pi / 400$, respectively, with the carrier at an angular frequency of $2 \pi / 1024$. The demodulated data are shown in Fig. 11(b). The bright regions are avidin, and the dark regions are blank biotin-conjugated PSI. The demodulation removes the high-frequency component of the carrier wave together with systematic errors caused by timing jitter and repositioning accuracy. This enables accurate differencing of data before and after incubation of a sample containing target molecules.

The precision of the optical system is tested by scanning a disk region consecutively, either with or

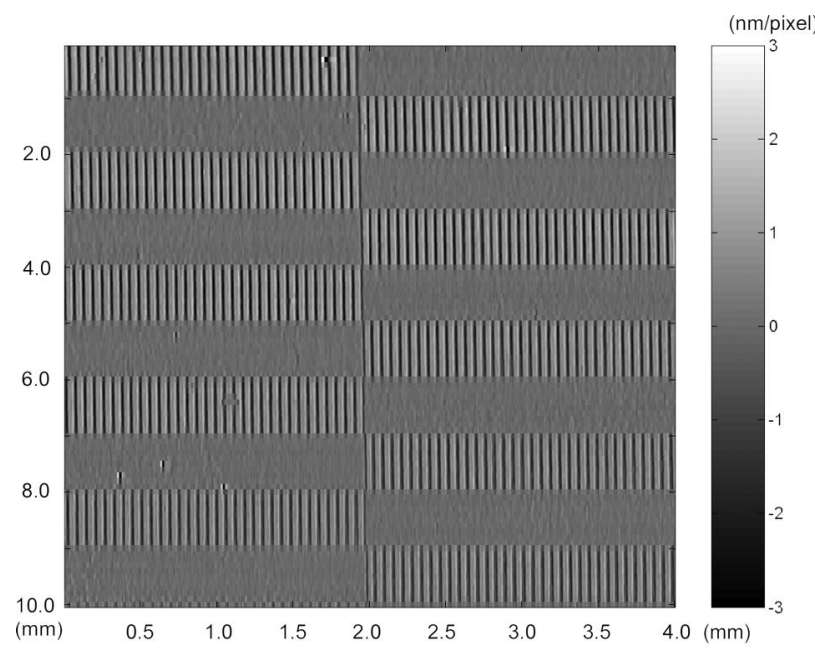

(a)

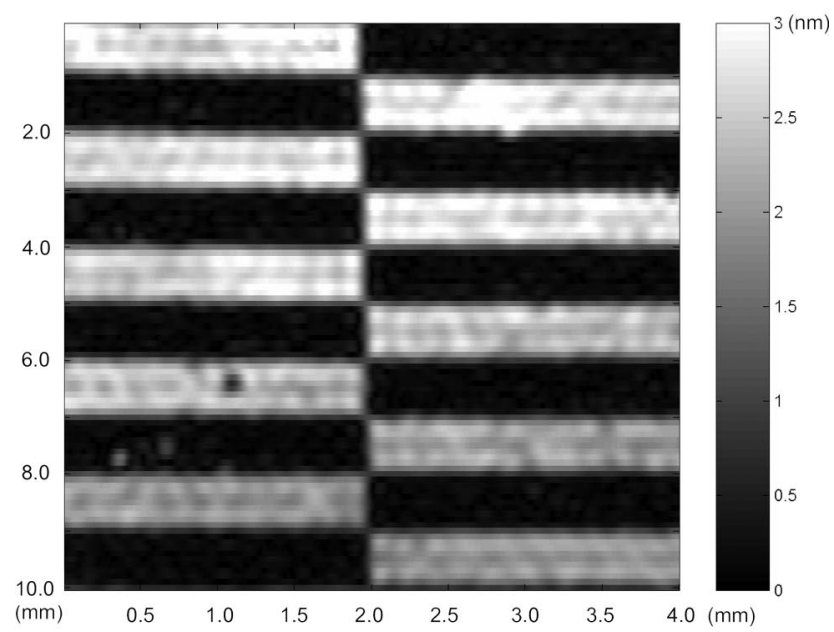

(b)

Fig. 11. Example of frequency sideband demodulation. The carrier frequency neighborhood is isolated in the frequency domain and transformed to dc and inverse transformed. Data on the top are the raw data. Data on the bottom represent the protein envelope function. The filter bandpass is approximately $70 \mu \mathrm{m}$.

without dismounting the disk, and calculating the differences between scans. When the disk is dismounted, the two data sets are registered by a mark on the outer rim of the disk. The histograms in Fig. 12 show the differences with and without dismounting. The demodulation process significantly reduces the error between scans by removing the high-frequency carrier wave of the signal. Under assay-like conditions (with demodulation and with a dismount to perform incubation), the repeatibility of the consecutive scans is $90 \mathrm{pm}$. In the best-case scenario (with demodulation and no dismount), the repeatability of consecutive scans is $20 \mathrm{pm}$. Note that the baseline in the power spectrum in Fig. 10(b) is at 100 pm compared to $20 \mathrm{pm}$ from the data in Fig. 12. These two measurements measure different things. The baseline of the power spectrum measures the meansquared surface roughness. This surface roughness is not "noise" because it is physically repeatable and 


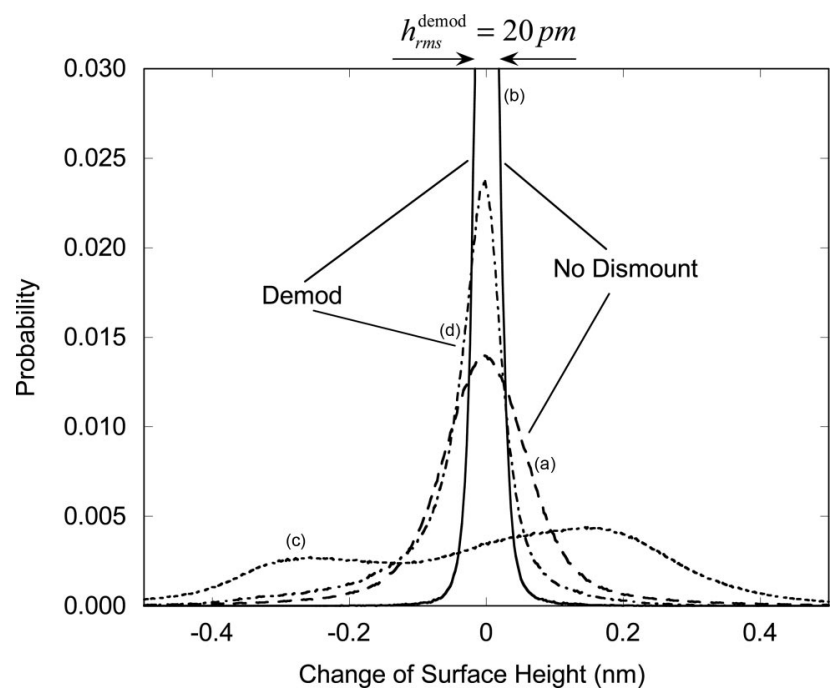

Fig. 12. Histograms of the pixel differences (expressed in $\mathrm{nm}$ ) between two successive scans. Curves are for four different conditions: (a) straight subtraction of the direct topology without dismounting, (b) subtraction of the data in (a) after demodulation, (c) straight subtraction with a dismount and remount of the disk, and (d) the same as (c) but with demodulation. Demodulation improves the accuracy of successive scans, while dismounting degrades it. The practical limit for an assay is curve (d) with a width of $90 \mathrm{pm}$.

measurable for each successive rotation of the disk. It is the variance of the surface roughness measurements that equals $20 \mathrm{pm}$.

\section{Scaling Surface Mass Sensitivity}

To compare the performance of the PC-class BioCD (obtained as 20 pm surface height resolution in Fig. 12) to the sensitivity of other sensing techniques, such as surface plasmon resonance that is expressed as mass per area, the minimum detectable height per pixel needs to be scaled to take into account averaging of the signal over area. The scaling of the mass sensitivity is performed by assuming that the scanning error follows an uncorrelated random distribution so that by averaging over an area that is $N$ times larger the standard error of the measurement of the surface dipole density is reduced by $\sqrt{N}$. The equivalent scaled minimum measurable surface height when averaged over a scale $L$ is given by

$$
\Delta h_{L}=\Delta h_{\text {meas }} \sqrt{\frac{w_{\text {meas }}^{2}}{A}}=\Delta h_{\text {meas }} \frac{w_{\text {meas }}}{L},
$$

where $A$ is the total measurement area, the scale $L$ $=\sqrt{A}$, and $w_{\text {meas }}^{2}$ is the effective measurement area associated with a single data point. The minimum detectable mass associated with this protein height is

$$
\Delta m_{L}=\Delta h_{L} \rho_{m} A=\Delta h_{\text {meas }} \rho_{m} w_{\text {meas }} L .
$$

Because of the scale-dependence on $L$ in Eq. (22), there is no intrinsic surface mass density for detec- tion. This means that the smallest surface mass density that can be measured depends on the area over which the data are averaged. On the other hand, there is an intrinsic property associated with the detection sensitivity, which is obtained by dividing the mass in Eq. (22) by the linear scale over which the data are averaged. This leads to an intrinsic scaling mass sensitivity given by

$$
S=\frac{\Delta m_{L}}{\sqrt{A}}=\rho_{m} \Delta h_{\text {meas }} w_{\text {meas }},
$$

which has the units of mass per length. From this sensitivity, the scale-dependent minimum detectable surface mass density is given by

$$
\sigma_{\min }=\frac{S}{\sqrt{A}}
$$

in units of grams per area, and the scale-dependent minimum captured mass that can be detected from that assay is given by

$$
\Delta m_{A}=S \sqrt{A}
$$

in units of grams.

Under the conditions of Fig. 12 leading to $\Delta h_{\text {meas }}$ $=20 \mathrm{pm}$, the demodulation procedure acts as an effective low-pass filter. The data rate is $1 \mathrm{Msamp} / \mathrm{s}$ for $1 \mu \mathrm{s}$ per data point. At a radius of $r=30 \mathrm{~mm}$, and an $80 \mathrm{~Hz}$ spin frequency, the tangential velocity is $15 \mathrm{~m} / \mathrm{s}$. The length swept out at this velocity in $1 \mu \mathrm{s}$ is $15 \mu \mathrm{m}$. In the sideband demodulation procedure, the bandwidth was chosen to be $60 \mathrm{kHz}$ centered on a carrier frequency of $80 \mathrm{kHz}$ generated by 1024 spokes of $100 \mu \mathrm{m}$ width. This gives an integrated length of $80 \times 100 \mu \mathrm{m} / 60=133 \mu \mathrm{m}$. In the radial direction, the bandwidth was half the radial frequency. With a radial pitch of $20 \mu \mathrm{m}$, this yields an integrated length of $40 \mu \mathrm{m}$. Therefore, the effective measurement area is $w_{\text {meas }}^{2}=40 \mu \mathrm{m} \times 133 \mu \mathrm{m}$ $=5.3 \times 10^{-3} \mathrm{~mm}^{2}$, and $w_{\text {meas }}=73 \mu \mathrm{m}$. The corresponding mass in this effective measurement area is $120 \mathrm{fg}$. Therefore, the scaling surface mass density is $S=\left(1 \mathrm{pg} / \mu \mathrm{m}^{3}\right)(20 \mathrm{pm})(73 \mu \mathrm{m})=1.5 \mathrm{pg} / \mathrm{mm}$, and the minimum detectable surface mass density at a scale of $1 \mathrm{~mm}$ is $\sigma_{\mathrm{mm}}=1.5 \mathrm{pg} / \mathrm{mm}^{2}$.

The scaling surface mass sensitivity is derived from an experimental quantity $\left(\Delta h_{\text {meas }}=20 \mathrm{pm}\right)$ obtained under ideal experimental conditions without dismounting the disk. Under the conditions of an assay, the disk is dismounted during the incubation and placed in solution, sometimes for many hours. This causes background changes in the protein spots and in the land surrounding the spots. Under these assay conditions, the minimum detectable surface height increases about fivefold to $90 \mathrm{pm}$, which increases the scaling mass sensitivity to approximately $7 \mathrm{pg} / \mathrm{mm}^{2}$. This $\mathrm{mm}$-scale sensitivity is comparable to typical values determined by SPR [4]. This sensi- 
tivity is gained without the need for resonance and hence is more robust and easy to manufacture than other interferometric approaches or resonance approaches that rely on resonance to provide high sensitivity.

\section{IgG Reverse Immunoassay}

To demonstrate the potential of using phase-contrast BioCDs as a platform for an immunoassay, we performed reverse assays to test the performance of the system using two different immobilization methods. A reverse assay immobilizes the antigen on the disk, which captures antibodies out of solution. We performed two different assays. In the first assay, the protein was patterned using gel printing, and the solution containing the analyte was applied across broad sectors separated by hydrophobic barriers. In the second assay, the protein was patterned using photolithography, and the solution containing the analyte was applied in spots.

In the first assay, the disk was gel-printed with Fluorescein- (FITC-) BSA in a 1024-radial-spoke pattern. The widths of the spokes increased with radius and were approximately $100-200 \mu \mathrm{m}$ wide to maintain a 50\% duty cycle at different radii. After the gel printing, the disk was divided into four quadrants by polydimethylsiloxane (PDMS) hydrophobic barriers. The four quadrants were backfilled separately with solutions of: 1) Mouse IgG, 2) FITC-BSA, 3) Horse IgG, and 4) $10 \mathrm{mM}$ PBS buffer at pH 7.4, respectively. The proteins in the solutions bind to the active surfaces between the printed BSA spokes, and form a periodic spoke pattern alternating between BSA and the specific IgG antigens. The protein solutions for this second printing step had a concentration of $10 \mu \mathrm{g} / \mathrm{mL}$ in PBS buffer. After the printing the disk was washed in DI water for $2 \mathrm{~min}$. Next came the incubation of the disk with sample containing analyte. The disk was incubated in two separate bands with solution containing antibody molecules. The inner band was incubated with antimouse IgG, and the outer band was incubated with antihorse IgG, both at a concentration of $10 \mu \mathrm{g} / \mathrm{mL}$ in PBS buffer. A central band remained blank to separate the two incubation bands. The disk was incubated for $30 \mathrm{~min}$ and then washed in DI water for 2 min.

In the second assay, the disk was patterned photolithographically beginning with a surface functionalized by APTES followed by photoresist that was patterned by photolithography. After the photoresist was exposed and developed, the exposed surface was passivated by sodium borohydride, as described previously, and the photoresist was removed. This process established a spatially repeating pattern of activated surface chemistry alternating with passivated surface. Onto this active patterned surface, $200 \mu \mathrm{L}$ drops of $10 \mu \mathrm{g} / \mathrm{mL}$ of Mouse IgG and Horse IgG were spotted separately and stood for $30 \mathrm{~min}$ before washing. The resulting spot size was approximately $10 \mathrm{~mm}$. The protein attaches covalently to the active surface, but not to the passivated surface. This produced a periodic protein pattern of IgG alter-

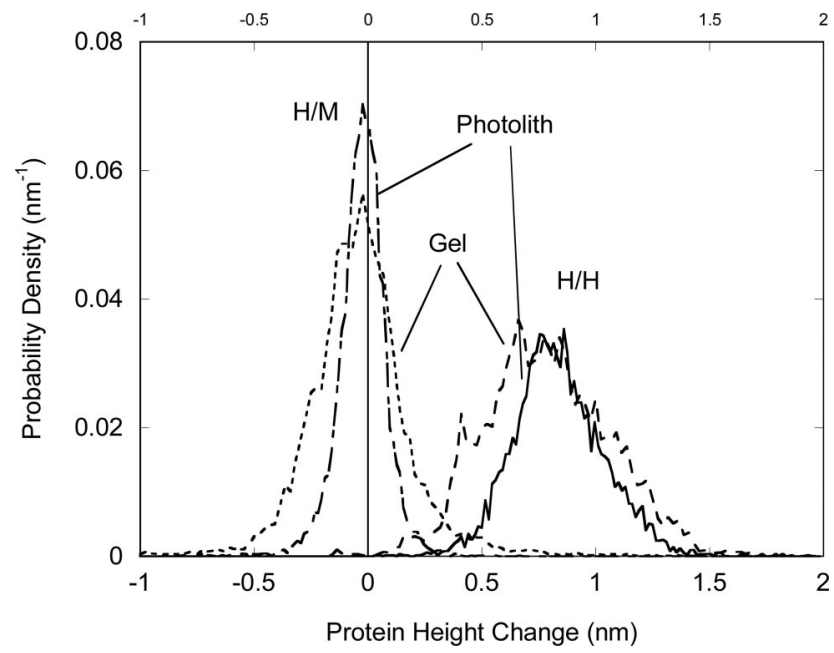

Fig. 13. Histogram of protein height change for two assays each performed at $10 \mu \mathrm{g} / \mathrm{ml}$ analyte concentration in PBS using two immobilization techniques: gel print and photolithography. Specific assay is horse-antihorse. Negative control was mouseantihorse.

nating with land. The printed disk was then incubated for 30 min using $200 \mu \mathrm{L}$ drops of antihorse or antimouse IgG spotted around the disk at increasing concentrations from $100 \mathrm{ng} / \mathrm{mL}$ to $10 \mu \mathrm{g} / \mathrm{mL}$ in PBS.

The protein height change is obtained by a direct subtraction of the measured surface height before and after incubation by the solutions containing antibodies. Histograms of the height change are shown in Fig. 13 for the photolithographic assay and for the gel-print assay, both at analyte concentrations of $10 \mu \mathrm{g} / \mathrm{ml}$ in PBS. There are two distributions for each assay: a cross-reactivity assay in which horse antibody is presented to printed mouse antigen as the negative control, and a specific assay in which horse antibody in solution is presented to printed horse antigen. The distribution for the gel-printed assay is noticeably broader than that of the photolithographically-printed

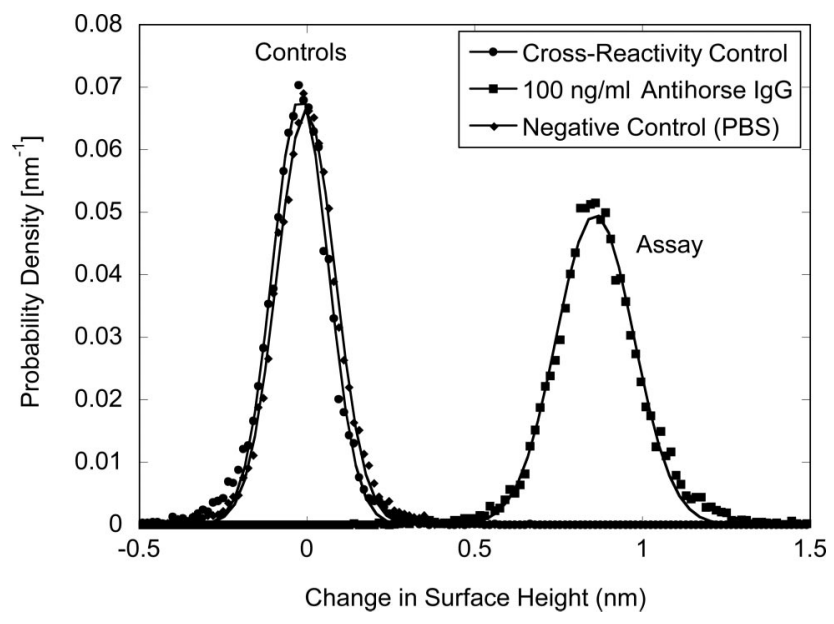

Fig. 14. Histogram of the disk height change in response to a $100 \mathrm{ng} / \mathrm{ml}$ assay compared against a negative control with PBS solution and a cross-reactivity control with horse incubated against mouse antigen. 
assay because of a lower print homogeneity. The two distributions are clearly separated between the controls and the specific assays.

These assays are saturated because typical equilibrium constants for immunoassays are in the range of $1-10 \mathrm{ng} / \mathrm{ml}$, which are well below the concentrations used here. The saturated character of these assays is further borne out in the height change histograms shown in Fig. 14 for the $100 \mathrm{ng} / \mathrm{ml}$ assay that was performed on the photolithographically printed disk. In this figure, an additional negative control (disk incubated with PBS solution with no protein) is shown compared with the $100 \mathrm{ng} / \mathrm{ml}$ antihorse-on-horse assay. At $100 \mathrm{ng} / \mathrm{ml}$, the overlap between the positive and the negative assays is only $0.2 \%$, corresponding to $0.2 \%$ false positives and false negatives.

\section{Discussion}

Laser scanning on a spinning biochip has several advantages that make this readout method a sensitive optical biosensor. The most important advantage is the $50 \mathrm{~dB}$ suppression of the noise floor by operating at a high detection frequency far from $1 / f$ noise. A second practical advantage is the ability to repeatedly measure the same spot many times on successive cycles to perform signal averaging. The high speed of the disk makes the read time practical despite the large areas covered. With a focal spot diameter of $20 \mu \mathrm{m}$ and a pitch of $20 \mu \mathrm{m}$, and using eight averages per track, a BioCD can be read out in less than an hour. During this time approximately 100 $\times 10^{6}$ optical measurements are made.

Another important feature of the BioCD is the quantitative nature of interferometry, in contrast to semiquantitative approaches such as the EnzymeLimited Immunosorbent Assay (ELISA). Interferometry is capable of subpicometer surface height metrology in the shot-noise limit. Although our application is limited by surface roughness, we still achieve a surface height repeatability of $20 \mathrm{pm}$ averaged over approximately $70 \mu \mathrm{m}$ to achieve a scaling surface mass sensitivity of $1.5 \mathrm{pg} / \mathrm{mm}$. An important result of this paper is this identification of an intrinsic property of the disk and system, which has units of mass per length. Most mass sensitivities are quoted in units of mass per area, which is not an intrinsic property, but changes as the area over which the data are averaged changes. By defining the scaling mass sensitivity correctly, it is used to calculate the massper-area sensitivity at other scales. For instance, at $1 \mathrm{~mm}$, our mass sensitivity is $1.5 \mathrm{pg} / \mathrm{mm}^{2}$, which compares favorably with sensitivities of SPR. It is important to state here that we have achieved even higher surface mass sensitivity down to $0.25 \mathrm{pg} / \mathrm{mm}$ using a different interferometric quadrature detection approach on the BioCD [29], and even further improvements are anticipated.

The immunological assays we performed on the phase-contrast BioCD were all performed under high analyte concentrations that drove the assays to sat- uration. Because the interferometric measurement is quantitative, it can be used at lower concentrations to obtain a dose response curve. The saturated height increase was only about $1 \mathrm{~nm}$ in all cases, which is only about $10 \%-20 \%$ of a monolayer thickness for a dense antibody coverage of the surface. This may have several causes, the most severe cause being that some protein denatures during disk dry-downs rendering some of the antigen or some of the antibodies inactive. We are currently working with disk preparations that never allow the printed proteins to dry out before being exposed to sample containing analytes.

In conclusion, we have demonstrated a new quadrature class of the BioCD called the differential-phasecontrast-class. It has simple disk fabrication and simple far-field optics, and has achieved a surface height sensitivity down to $20 \mathrm{pm}$. The surface mass sensitivity is comparable to other label-free approaches, but is achieved with greater simplicity that lends itself to scaling up to many analytes per disk.

This work was supported by sponsored research from QuadraSpec, Inc., through the Purdue Research Foundation.

\section{References}

1. G. Gauglitz, "Direct optical sensors: principles and selected applications," Anal. Bioanal. Chem. 381, 141-155 (2005).

2. R. Cush, J. M. Cronin, W. J. Stewart, C. H. Maule, J. Molloy, and N. J. Goddard, "The resonant mirror-a novel optical biosensor for direct sensing of biomolecular interactions 1. Principle of operation and associated instrumentation," Biosens. Bioelectron. 8, 347-353 (1993).

3. M. P. DeLisa, Z. Zhang, M. Shiloach, S. Pilevar, C. C. Davis, J. S. Sirkis, and W. E. Bentley, "Evanescent wave long period fiber Bragg grating as an immobilized antibody biosensor," Anal. Chem. 72, 2895-2900 (2000).

4. J. Homola, S. S. Yee, and G. Gauglitz, "Surface plasmon resonance sensors: review," Sens. Actuators B 54, 3-15 (1999).

5. V. S. Y. Lin, K. Motesharei, K. P. S. Dancil, M. J. Sailor, and M. R. Ghadiri, "A porous silicon-based optical interferometric biosensor," Science 278, 840-843 (1997).

6. C. Striebel, A. Brecht, and G. Gauglitz, "Characterization of biomembranes by spectral ellipsometry, surface-plasmon resonance, and interferometry with regard to biosensor application," Biosens. Bioelectron. 9, 139-146 (1994).

7. N. Ramachandran, D. N. Larson, P. R. H. Stark, E. Hainsworth, and J. LaBaer, "Emerging tools for real-time label-free detection of interactions on functional protein microarrays," FEBS J. 272, 5412-5425 (2005).

8. F. Prieto, B. Sepulveda, A. Calle, A. Llobera, C. Dominguez, and L. M. Lechuga, "Integrated Mach-Zehnder interferometer based on ARROW structures for biosensor applications," Sens. Actuators B 92, 151-158 (2003).

9. G. H. Cross, A. Reeves, S. Brand, M. J. Swann, L. L. Peel, N. J. Freeman, and J. R. Lu, "The metrics of surface adsorbed small molecules on the Young's fringe dual-slab waveguide interferometer," J. Phys. D 37, 74-80 (2004).

10. P. I. Nikitin, B. G. Gorshkov, E. P. Nikitin, and T. I. Ksenevich, "Picoscope, a new label-free biosensor," Sens. Actuators B 111, 500-504 (2005).

11. J. Dyson, "Common-path interferometer for testing purposes," J. Opt. Soc. Am. 47, 386-390 (1957).

12. J. Gluckstad and P. C. Mogensen, "Optimal phase contrast in common-path interferometry,” Appl. Opt. 40, 268-282 (2001). 
13. C. Chien, S. Jenn-Chyang, H. Yeu-Chuen, and Y. Chen-Kee, "Common-path optical heterodyne profilometer: a configuration,” Appl. Opt. 37, 4137-4142 (1998).

14. C. M. Feng, Y. C. Huang, J. G. Chang, M. Chang, and C. Chou, "A true phase-sensitive optical heterodyne polarimeter on glucose concentration measurement," Opt. Commun. 141, 314321 (1997).

15. R. Jenison, S. Yang, A. Haeberli, and B. Polisky, "Interferencebased detection of nucleic acid targets on optically coated silicon," Nat. Biotechnol. 19, 62-65 (2001).

16. M. V. Sarunic, S. Weinberg, and J. A. Izatt, "Full-field sweptsource phase microscopy," Opt. Lett. 31, 1462-1464 (2006).

17. M. M. Varma, H. D. Inerowicz, F. E. Regnier, and D. D. Nolte, "High-speed label-free detection by spinning-disk microinterferometry," Biosens. Bioelectron. 19, 1371-1376 (2004).

18. B. Cunningham, P. Li, and J. Pepper, "Colorimetric resonant reflection as a direct biochemical assay technique," Sens. Actuators B 81, 316-328 (2002).

19. C. W. See, M. V. Iravani, and H. K. Wickramasinghe, "Scanning differential phase-contrast optical microscope-application to surface studies," Appl. Opt. 24, 2373-2379 (1985).

20. A. J. Pidduck, D. J. Robbins, D. B. Gasson, C. Pickering, and J. L. Glasper, "In situ laser light scattering. II. Relationship to silicon surface topography," J. Electrochem. Soc. 136, 30883094 (1989).

21. M. Varma, D. D. Nolte, H. D. Inerowicz, and F. E. Regnier,
"Multianalyte array microdiffraction interferometry," Proc. SPIE 4626, 69-77 (2002).

22. M. M. Varma, D. D. Nolte, H. D. Inerowicz, and F. E. Regnier, "Spinning-disk self-referencing interferometry of antigenantibody recognition," Opt. Lett. 29, 950-952 (2004).

23. L. Peng, M. Varma, H. D. Inerowicz, F. E. Regnier, and D. D. Nolte, "Adaptive optical BioCD for biosensing," Appl. Phys. Lett. 86, 183902 (2005).

24. D. D. Nolte, T. Cubel, L. J. Pyrak-Nolte, and M. R. Melloch, "Adaptive beam combining and interferometry using photorefractive quantum wells,” J. Opt. Soc. Am. B 18, 195-205 (2001).

25. D. D. Nolte, Photorefractive Effects and Materials (Kluwer Academic, 1995).

26. C. W. See, R. K. Appel, and M. G. Somekh, "Scanning differential optical profilometer for simultaneous measurement of amplitude and phase variation," Appl. Phys. Lett. 53, 10-12 (1988).

27. M. R. Atkinson, A. E. Dixon, and S. Damaskinos, "Surfaceprofile reconstruction using reflection differential phasecontrast microscopy,” Appl. Opt. 31, 6765-6771 (1992).

28. C. Chien, L. Chung-Wei, and P. Li-Cheng, "Polarized differential-phase laser scanning microscope," Appl. Opt. 40, 95-99 (2001).

29. D. D. Nolte and M. Zhao, "Scaling mass sensitivity of the BioCD at $0.25 \mathrm{pg} / \mathrm{mm}$," Proc. SPIE 6380, 63800J (2006). 